\title{
CHANGES IN THE HETEROGAMETIC MECHANISM OF SEX DETERMINATION
}

\author{
JAMES J. BULL* and ERIC L. CHARNOV \\ Department of Biology, University of Utah, Salt Lake City, Utah 84112
}

Received 23.iv.76

\section{SUMMARY}

This paper attempts to explain how the different heterogametic mechanisms of sex determination, such as male and female heterogamety, can arise from a single ancestor. We study this problem by first building and analysing some models and then comparing the models to relevant data from animals. Based on these comparisons, the models appear to be useful for understanding how sex determining mechanisms evolve. Some results of the models include:

(1) A mutant sex determiner invading a population of male or female heterogamety creates the potential for the evolution of a new heterogametic sex determining mechanism.

(2) Certain mutants will lead to a new system of male heterogamety whereas others will lead to a new system of female heterogamety.

(3) These changes take place through an intermediate population characterised by multigenic sex determination. In some cases the multigenic mechanism is stable and will not revert to male or female heterogamety. The success of the invading mutant depends upon both (i) the relative viabilities of the different genotypes, and (ii) the segregation ratio of the $\mathrm{X}$ and $\mathrm{Y}$ in males; the population primary sex ratio is otherwise maintained at $1 / 2$.

(4) The models show how the different systems of genic balance arise and how sex determiners can become inherited autosomally.

\section{Introduction}

SEx determination among many animals is achieved by a "simple" mechanism of heterozygosity in one sex (heterogamety) and homozygosity in the other. Either the male or the female may be heterogametic, and these two heterogametic systems are known for a wide variety of animal groups (White, 1973). The observations from higher vertebrates imply that changes in the heterogametic sex occur infrequently (White, 1973); data from poeciliid fishes (Kallman, 1965) and some dipterans (Martin, 1966) suggest that the system often changes because male and female heterogamety are sometimes found among closely related species and even within the same population. A problem emerges from these observations: how does natural selection act upon changes in the sex determining system?

This problem was initially posed by Winge $(1932,1934)$ who was able to select for a change in the sex determining mechanism of his guppies. Winge suggested that autosomal sex determiners are responsible for overthrowing the inheritance of the sex chromosomes. Although there has been a lot of experimental work on this problem since Winge (see Kallman, 1965, 1968) and some theoretical work on different kinds of sex determining systems (Scudo, 1964, 1967), no genetical study has considered how the simple mechanisms of heterogametic sex determination evolve. In this paper we present a set of one- and two-loci models that provide a way of interpreting changes in simple heterogametic sex determining systems.

* Present address: Dept. of Genetics, University of Wisconsin, Madison, Wisconsin 53706.

$$
39 / 1-A
$$


These models are compared to available data from animals in an attempt to evaluate their validity.

\section{THE MODELS}

Male and female heterogamety are similar in that both systems give rise to homozygotes of one sex and heterozygotes of the other. The basis for determining which sex is heterozygous should depend upon the balance between male and female determining factors in the heterozygote. The homogametic sex is homozygous for all sex determiners; therefore, the haploid constitution of this sex, $\mathrm{X}+\mathrm{A}$, must have the same sex determining effect as the diploid constitution, $\mathrm{XX}+\mathrm{AA}$, where $\mathrm{A}$ is a haploid set of autosomes. The heterogametic sex $(\mathrm{XY}+\mathrm{AA})$ must have the opposing and stronger sex determining factors in $\mathrm{Y}+\mathrm{A}$ than those in $\mathrm{X}+\mathrm{A}$. Thus, if the male is heterogametic, $\mathrm{Y}+\mathrm{A}$ must be male determining and stronger than $\mathrm{X}+\mathrm{A}$ which is female determining. If the female is heterogametic, then $\mathrm{Y}+\mathrm{A}$ (also known as $\mathrm{W}+\mathrm{A}$ ) must be female determining and stronger than the male determining $\mathrm{X}+\mathrm{A}(\mathrm{Z}+\mathrm{A})$.

\begin{tabular}{|c|c|c|c|}
\hline & & & \\
\hline Strongly & $\begin{array}{l}\text { M } \\
\text { termining }\end{array}$ & Mildly ${ }_{\mathrm{r}}$ & $\begin{array}{l}\text { M } \\
\text { ermining }\end{array}$ \\
\hline$q$ & $\sigma^{*}$ & 우 & $\sigma^{*}$ \\
\hline $\mathrm{Xx}$ & $\mathrm{XY}$ & $\mathrm{xX}$ & $\mathrm{XY}$ \\
\hline & $\mathrm{XM}$ & $\mathrm{XM}$ & MM \\
\hline Conditions: & )$>q(\mathbf{X}+\mathbf{A})$ & $q(\mathrm{X}+$ & $\mathrm{M}+\mathrm{A})$ \\
\hline & & & \\
\hline Strongly $\mathrm{f}$ & $\begin{array}{l}\text { F } \\
\text { etermining }\end{array}$ & Mildly fe & $\begin{array}{l}\mathrm{F} \\
\text { termining }\end{array}$ \\
\hline$q$ & $\sigma$ & q & $\sigma$ \\
\hline $\mathrm{XX}$ & $X Y$ & $\mathrm{XX}$ & $X Y$ \\
\hline FX & & FX & \\
\hline FY & YY & FF & FY \\
\hline Conditions: & )$>\sigma^{-1}(Y+A)$ & $\sigma(\mathrm{Y}+$ & $\mathrm{F}+\mathrm{A})$ \\
\hline
\end{tabular}

Sex determining mutants arising at the existing sex determining locus. Each of these has a counterpart in the two-loci models. "Locus" means any region which assorts from a heterozygote as a unit. Each model, A, B, C, or D, lists the male and female genotypes in the population which result from the presence of the specified mutant ( $M$ or $F$ ). Each model is arranged so that the top line is the recurrent pair for male heterogamety (ancestral). All genotypes below the top line are those that result from the presence of the mutant. The bottom line represents those genotypes of the new recurrent pair. The conditions listed below each column are the requirements for the strength of the mutant, where A represents a haploid set of autosomes. " $O(W+A)>$ " is read " the female determining strength of W+A is greater than ...". This notation also implies that the quantity in the brackets has a net effect of the symbol outside. (B) and (C): the two mutants which lead to female heterogamety in a population originally of pure male heterogamety. (A) and (D): mutants maintaining the existing system of male heterogamety. (A) and (D) may simply be thought of as normal variations among the existing $X$ and $Y$ regions, including translocations to autosomes. In (D) the equilibria for female frequencies are given by the Hardy-Weinberg function in terms of the male frequencies. Since (A) and (D) are rather straightforward, they are given very little attention elsewhere in the paper. 
We have used the following approach to understand how the sex determining mechanisms may change. A mutant gene with sex determining properties is introduced into a population of simple male heterogamety. The mutant causes a change in the inheritance of the $\mathrm{X}$ and $\mathrm{Y}$ sex determiners, and this gives rise to additional genotypes in the population. The fate of the established sex determining mechanism depends upon the set of new genotypes and the way that selection acts upon these in the population. Our results are based on computer simulations and, in some cases, algebraic analyses.

The set of genotypes resulting from the mutant depends upon three factors: (1) the locus of introduction, (2) how the sex determiners interact to produce one sex phenotype or the other, and (3) the strength of the mutant relative to the established $\mathrm{X}, \mathrm{Y}$ and autosomal sex determiners.

(1) We have considered models in which the mutant is introduced at the $\mathrm{X}-\mathrm{Y}$ locus (single locus, table 1 ) and models in which the mutant is introduced at a locus not linked to the X-Y locus (two loci, tables 2 and 3). In these models, the X, Y, and mutant may be treated as a single-gene locus or entire chromosomes that are completely linked. The mutant may arise by mutation or by translocation of an existing sex determiner.

(2) It was assumed that the sex determiners operated collectively in a simple dominance-recessive manner; when the aggregate of male determiners was stronger than the aggregate of female determiners, a male phenotype resulted and vice versa. This is Bridges' theory of genic balance

TABLE 2

A

Mutant F Strongly female determining

\begin{tabular}{|c|c|c|c|}
\hline \multicolumn{2}{|c|}{ 우 } & \multicolumn{2}{|c|}{ 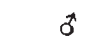 } \\
\hline bb & XX & $\mathrm{bb}$ & $X Y$ \\
\hline $\mathrm{bF}$ & $\mathrm{XX}$ & & \\
\hline $\mathrm{bF}$ & $X Y$ & & \\
\hline $\mathrm{bF}$ & $Y Y$ & $\mathrm{bb}$ & $Y Y$ \\
\hline
\end{tabular}

Conditions: $P(\mathrm{bF}+\mathrm{AA})>$ $\sigma^{*}(\mathrm{YY}+\mathrm{AA})$

$\mathrm{C}_{1}$
B

Mutant M

Mildly male determining

\begin{tabular}{|c|c|}
\hline 우 & 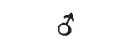 \\
\hline bb $\mathrm{XX}$ & $\begin{array}{l}\text { bb XY } \\
\text { bM XY } \\
\text { MM XY }\end{array}$ \\
\hline bM XX & $\mathrm{MM} \mathrm{XX}$ \\
\hline
\end{tabular}

$\sigma(\mathrm{MM}+\mathrm{AA})>q(\mathrm{XX}+\mathrm{AA})>$ $\sigma(\mathrm{bM}+\mathrm{AA})$

$\mathrm{C}_{2}$

Mutant $F$, weakly female determining

\begin{tabular}{|c|c|c|c|c|}
\hline & 우 & $0^{\pi}$ & q & 屯 \\
\hline $\mathrm{bb}$ & $\mathbf{X X}$ & bb $X Y$ & bb XX & bb $X Y$ \\
\hline $\mathrm{bF}$ & $\mathrm{XX}$ & $\mathrm{bF} X Y$ & bF $X X$ & $\mathrm{bb} \quad \mathrm{YY}$ \\
\hline FF & $\mathrm{XX}$ & bF YY & bF $X Y$ & $b F \quad Y Y$ \\
\hline FF & $\mathrm{XY}$ & FF YY & FF $\quad X Y$ & FF YY \\
\hline \multicolumn{3}{|c|}{$\begin{array}{c}\delta^{t}(\mathrm{YY}+\mathrm{AA})>\rho(\mathrm{FF}+\mathrm{AA})> \\
\delta^{\prime}(\mathrm{XY}+\mathrm{AA})>q(\mathrm{bF}+\mathrm{AA})\end{array}$} & \multicolumn{2}{|c|}{$\begin{array}{r}d(\mathrm{YY}+\mathrm{AA})>\mathcal{F}(\mathrm{FF}+\mathrm{AA})> \\
q(\mathrm{bF}+\mathrm{AA})>\delta^{\prime}(\mathrm{XY}+\mathrm{AA}\end{array}$} \\
\hline
\end{tabular}

Four kinds of mutations arising at a non-XY locus which lead to female heterogamety in a population originally of simple male heterogamety, $b b X X / b b X Y$. The mutant sex determiner arises at locus $\mathrm{b}$ which is not linked to the XY locus. Allele $\mathrm{b}$ has no sex determining influence. $C_{1}$ and $C_{2}$ both represent the general condition $\delta(Y Y+A A)>q(F F+$ $\mathrm{AA})>\delta(\mathrm{XY}+\mathrm{AA})$ and are not considered as distinct in the text. See table 1 for explanation of the format. 
and is the approach most commonly advocated by earlier workers (Bridges, 1925; Goldschmidt, 1934; Winge, 1937). Genic balance accounts for sex determination by the autosomes as well as the $\mathrm{X}$ and $\mathrm{Y}$. The $\mathrm{X}$ and $\mathrm{Y}$ are assumed to function only in sex determination, and only one of these, the $\mathrm{X}$ or $\mathrm{Y}$, need be sex determining. Since hermaphroditism is not considered in these models, our approach is of limited facility to sex determination in plants.

(3) The array of genotypes resulting from the mutant differed for mutants of different sex determining strength. A male determiner, $M$, being introduced at a locus (b) not linked to the X-Y locus, must be " strong enough " to override the femaleness of XX + AA. 'Two possibilities exist: (1) bM XX is male, or (2) bM XX is female and MM XX is male (tables $3 \mathrm{~A}$ and $2 \mathrm{~B}$, respectively). Allele " $\mathrm{b}$ " has no effect.

TABLE 3

A

Mutant M, strongly male determining

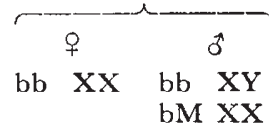

Conditions: $\delta(\mathrm{bM}+\mathrm{AA})>\rho(\mathrm{XX}+\mathrm{AA})$

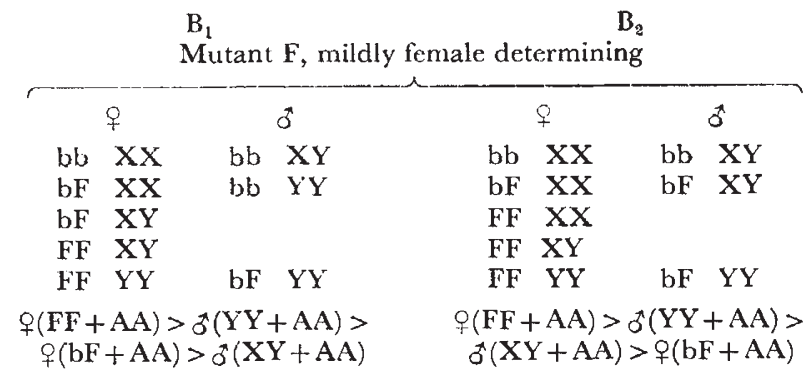

Three kinds of sex determining mutants arising at a non-XY locus which lead to a new system of male heterogamety in a population originally $b b X X / b b X Y . \quad B_{1}$ and $B_{2}$ both represent the general condition $Q(F F+A A)>\delta(Y Y+A A)>Q(b F+A A)$. Otherwise as in tablc 2 .

A female determining mutant, $\mathrm{F}$, faces a more complicated situation than a male determiner; $\mathrm{F}$ or FF must override the maleness of $\mathrm{XY}+\mathrm{AA}$. The mating of XY females to males creates YY individuals, which can be of either sex, depending upon the mutant. The cases to consider for a female determining mutant are the following: (3) bF YY is female (2A), (4) bF YY is male, $\mathrm{FF} Y Y$ is female, and $\mathrm{bF} \mathrm{XY}$ is male $\left(3 \mathrm{~B}_{2}\right)$ or female $\left(3 \mathrm{~B}_{1}\right)$; (5) FF $\mathrm{YY}$ is male, $\mathrm{FF} \mathrm{XY}$ is female, and $\mathrm{bF} \mathrm{XY}$ is male $\left(2 \mathrm{C}_{1}\right)$ or female $\left(2 \mathrm{C}_{2}\right)$. The models in which the mutant arises at the $\mathrm{X}-\mathrm{Y}$ locus are simpler than these (table 1).

If a sex determining mutant which is weaker than those considered above is introduced into a population, it will not alone cause a change in the inheritance of the $\mathrm{X}$ and $\mathrm{Y}$.

We have used the term "simple" heterogamety to denote the sex determining system in which a population consists of only one male and one 
female genotype, the male or female being heterozygous. This pair of genotypes, one male and one female, has the property that, when crossed, it gives rise to zygotes of these genotypes only. A male and a female genotype with this property are referred to as a recurrent genotype pair.

Inspection of tables 1-3 reveals that each mutant gives rise to one or more new genotypes. In all of the derived populations the inheritance of the $\mathrm{X}$ and $\mathrm{Y}$ has changed and sex is determined by more than just the $\mathrm{X}$ and Y. These sex determining mechanisms are termed multigenic and denote any population in which sex is decided by more than two sex determiners, although sex in an individual may still be decided by only two. The term "polygenic" has been used for sex determining mechanisms previously by Kosswig (1964) and Karlin and Feldman (1968a). A similar term, "polymeric", has been used by Bacci (1965) and "multifactorial" by Scudo (1967).

The models presented in this paper are part of a larger class of models which are called "incompatibility" models and which were studied by Fisher (1941), Bateman (1952), Finney (1952), Ewens (1964), Cannings (1968), Karlin and Feldman (1968a,b), and Falk and Li (1969). Scudo $(1964,1967)$ has presented models of mating systems which are equivalent to some of ours.

The behaviour of each model (except $1 \mathrm{~A}$ and 3A) was investigated by computer simulation. Equations for new genotype frequencies were derived by assuming random mating of all genotypes of one sex with those of the other sex in discrete generations with infinite population size. Thus, given a set of starting frequencies, the $F_{1}$ frequency of each genotype can be represented as a sum of parental mating combinations divided by the total number of offspring of that sex. All sex-specific factors (otherwise genotypeindependent) such as mortality and fertility cancel from the equations. Successive changes in genotype frequencies can be calculated on a computer for many generations. The two models not analysed by computer simulation ( $1 \mathrm{~A}$ and $3 \mathrm{~A}$ ) are the mathematical equivalent of a system analysed by others (Finney, 1952; Karlin and Feldman, 1968a). In addition to the numerical analyses, analytical solutions for equilibria were obtained for some of the models by setting the $F_{1}$ frequencies equal to the $\mathbf{P}_{\mathbf{1}}$ frequencies and solving the equations simultaneously.

\section{Results}

The introduction of a sex determining mutant creates a specific multigenic sex determining mechanism (tables 1-3). Each mechanism differs from the others in the male and female genotypes which are present, but there is an important similarity shared by all: there are two recurrent pairs in each model. One recurrent pair is the male and female genotype of the ancestral population of male heterogamety; the other is new. Therefore, each mutant has the potential to give rise to a new simple heterogametic mechanism in a population.

The mutant is always retained in the new recurrent pair and determines whether the male or female genotype of this pair is heterozygous. In some models the new recurrent pair is heterozygous in the female ( $1 \mathrm{~B}, \mathrm{C}, 2 \mathrm{~A}-\mathrm{D})$; in the other models the new recurrent pair is heterogametic in the male ( $\mathrm{A}, \mathrm{D}, 3 \mathrm{~A}-\mathrm{C})$. 
The analysis so far has focused on the genotypes which arise in a multigenic sex determining mechanism. It is also important to determine how selection acts on these genotypes and, thus, how a simple heterogametic sex determining mechanism can evolve from a multigenic one.

The models were initially analysed with the assumption that all genotypes within a sex had the same fitness. All behaved similarly according to a general rule: in each model there are an infinite number of neutral equilibria at which all genotypes may coexist. The population primary sex ratio of these equilibria is $1 / 2$. Scudo (1967) also observed the neutral equilibria. The frequencies of every genotype in the equilibrial set may be written as a function of the frequency of any one recurrent pair genotype (table 4, figs. 1 and 2). This set may be described as a continuous path of neutral equilibria from one recurrent pair to the other. Models $1 \mathrm{~A}$ and $3 \mathrm{~A}$ are unique because the equilibria are functions of just the male genotype frequencies; thus, all possible frequencies are equilibrial.

\section{TABLE 4}

In table $1 \mathrm{C}$ let the frequency of $\mathrm{YY}$ be $p$. The equilibrial set is given by

$$
\mathrm{XX}=\frac{(1-p)^{2}}{(1+p)^{2}}, \mathrm{FX}=\frac{2 p(1-p)}{(1+p)^{2}}, \mathrm{FY}=\frac{2 p}{1+p}, \mathrm{XY}=1-p
$$

In $2 \mathrm{~A}$ let the frequency of $\mathrm{bb} \mathrm{YY}$ be $p$. The equilibrial set is given by

$$
\mathrm{bbXX}=\frac{(1-p)^{2}}{(1+p)^{2}}, \mathrm{bFXX}=\frac{p(1-p)^{2}}{(1+p)^{2}}, \mathrm{bFXY}=\frac{2 p(1-p)}{1+p}, \mathrm{bFYY}=p, \mathrm{bbYY}=1-p
$$

Equilibrial conditions for models in $\mathrm{IC}$ and $2 \mathrm{~A}$. By appropriate choice of genotypes, the equilibrial equations in $\mathrm{BB}$ and $2 \mathrm{~B}$ are those for $1 \mathrm{C}$ and $2 \mathrm{~A}$ (respectively) with males and females interchanged. Similar equations to those of 2A are found in Scudo $(1964,1967)$. The equations for $2 \mathrm{C}$ and $3 \mathrm{~B}$ proved too complex to obtain. The graphs of these equations are plotted in fig. 1 .

The equilibrial set for most cases has been transformed on to twodimensional co-ordinate systems (figs. 1,2). These graphs provide the entire set of equilibrial frequencies. In $2 \mathrm{C}$ and $3 \mathrm{~B}$, for which analytical solutions were not obtained, graphs were constructed from sequential equilibria determined by computer analysis.

If initial genotype frequencies were not equilibrial, equilibrium was approached rapidly ( \pm 0.001 by 6 generations), the exact equilibrium depending upon the starting frequencies. Once an equilibrium was reached, the genotype frequencies remained at it in the absence of perturbation. Perturbation away from an equilibrium was followed by a return to an equilibrium, but not necessarily the same one. The stochastic processes of finite populations should introduce slight perturbations so that no single internal equilibrium would persist, and one recurrent pair would eventually become fixed.

The assumption of equal fitness of all genotypes may often be unreasonable. To get an indication of the effect of variation in fitness, the models were studied when the viability of a single genotype was altered. Again, the results from all models are similar and can be understood in the following way: (1) the differential-fitness models behaved like the equal fitness models in that the genotype frequencies initially approached a region in the former equilibrial set. However, once near the equilibrial path, (2) the genotype 
frequencies then began " migrating" along the path in one direction or the other toward a stable equilibrium. The sex ratio varies along this path, but is always close to $1: 1$. The effect of selecting against a genotype is to reduce its frequency to zero. The effect of increasing its fitness is to maximise
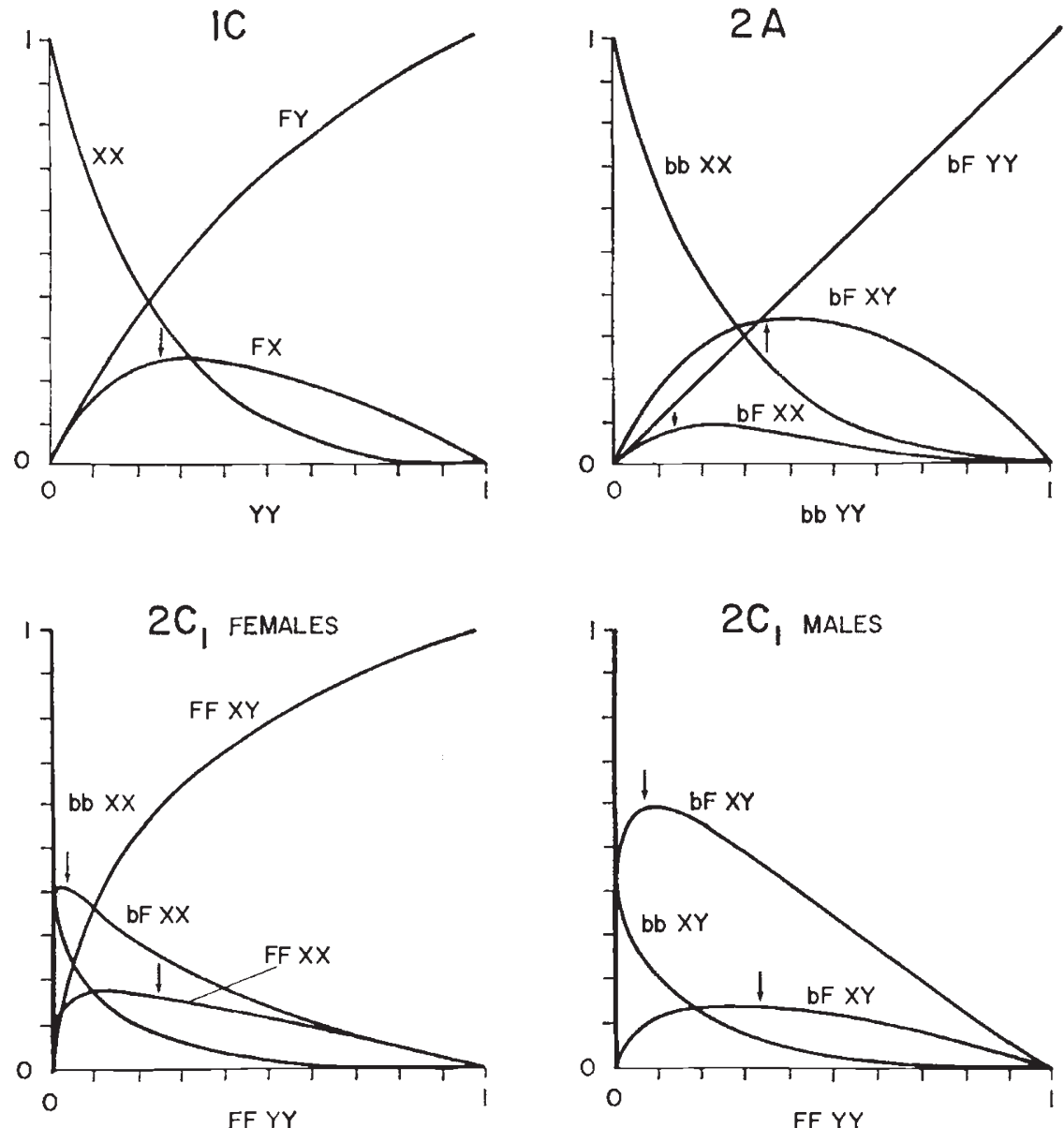

Fig.1 - Each graph corresponds to a model from one of the tables, as is indicated on the graph. The curves present neutral equilibrium values only (= viabilities) and do not give any information about the way that the system approaches an equilibrium. Given the frequency of the genotype indicated on the horizontal axis, the frequency of the other genotypes are given by the vertical distances to their respective curves. Arrow indicates the approximate position of the stable equilibrium when the genotype of the curve indicated has the higher viability. In models where only two genotypes of a sex occur, the graph (not shown) is the diagonal element from $(0,1)$ to $(1,0)$. The mating systems are equivalent (with sexes reversed) in $1 \mathrm{~B}$ and $1 \mathrm{C}, 2 \mathrm{~A}$ and $2 \mathrm{~B}$, and in $2 \mathrm{C}_{1}$ and $2 \mathrm{C}_{2}$; therefore, the graphs for one member of each pair are omitted.

its frequency to unity for any recurrent pair genotype. If the fitness of any recurrent genotype is altered, the effect is to establish that recurrent pair at fixation (increased fitness) or establish the other recurrent pair at fixation (decreased fitness).

In most models there are one or more genotypes that do not form a 
recurrent pair with any other of the opposite sex. The effect of fitness changes for one of these genotypes has different consequences. Inspection of the figures reveals that these genotypes have zero frequency at both end points and a maximum internal. Lowering the fitness of the genotype can lead to fixation of either endpoint, depending upon which side of this internal maximum the population frequencies initially fall : selection in these
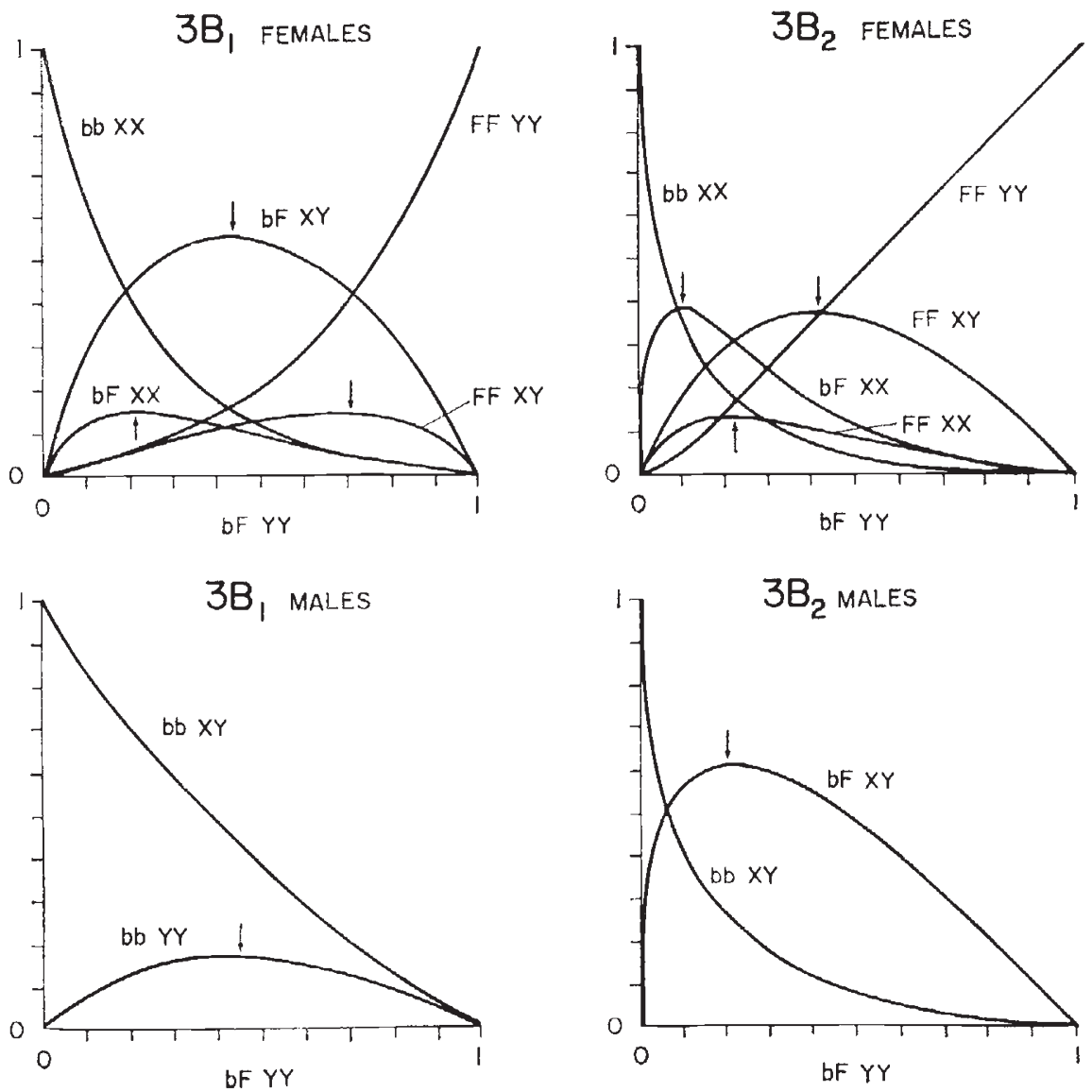

FIG. 2.-Graphs of the neutral equilibria values for the models in Table 3. Otherwise as in fig. 1.

cases would act against a rare mutant sex determiner. Increasing the fitness of this genotype leads to the establishment of a single equilibrium in the neighbourhood of the neutral equilibrium at which this genotype has maximum frequency. The equilibrium behaves as a stable one because it is approached from any point along the former equilibrial set. The positions of these stable polymorphisms (for a viability increase from 1.00 to 1.01 ) are indicated by arrows in the figures. For an intuitive, but superficial, similarity consider the behaviour of the Hardy-Weinberg system under heterozygote advantage. Due to the limitation of numerical analysis, we cannot rule out the possibility of other behaviours in these models.

The results may be summarised as follows. The introduction of a sex 
determining mutant into a population of male or female heterogamety alters the inheritance of the $\mathrm{X}$ and $\mathrm{Y}$. The resulting multigenic mechanism has the potential to change to a new system of heterogamety. This change is only likely to evolve when either genotype in the new recurrent pair has higher viability than the others, assuming a lower, but equal, viability among all others. Otherwise, the population will evolve "back" to the original system of heterogamety if one of the original genotypes has the highest viability, or evolve to a stable multigenic mechanism if the genotype with the highest viability is not a recurrent pair genotype.

\section{Comparing the models to natural populations}

(i) The levels of comparison

The basis of these models is largely theoretical; thus, some assessment of their relevance to natural populations is in order. Since the models may be valid approximations of natural changes in some ways but invalid in other ways, we discuss initially the different levels at which tests may be made and the implications of testing each level.

(1) The most basic part of the theory is that the introduction of a sex determiner into a population of male or female heterogamety provides the potential to evolve a new system of heterogamety through an intermediate mechanism of multigenic sex determination. Therefore, populations with multigenic sex determination should occur naturally and should also be reproducible under artificial conditions. These populations must have at least one new recurrent pair in order to bring about a change. If multigenic sex determining mechanisms are never observed and cannot be established artificially, then the approach presented here is probably invalid.

(2) The genotypes observed in any natural population with multigenic sex determination of one or two loci should correspond to one of the models in this paper. Systems with three and four loci should also be consistent with the assumptions used in building these models. The failure of populations to display the expected genotypes would indicate that the assumption of genic balance is in error. Alternative models assuming different interactions can be constructed easily.

(3) Complete assessment of the models requires comparison of observed genotype frequencies to the calculated equilibrial frequencies. Congruence between observed and expected provides the strongest evidence in favour of the model. If observed frequencies deviate greatly from any possible equilibrium it may suggest that the genotypes have been incorrectly assessed, or possibly, that the sex determiners are expressed inconsistently (e.g. partial penetrance). Ultimately, one would hope to have complete information on relative genotypic fitnesses to try and predict frequencies through successive generations.

\section{(ii) Natural examples}

Comprehensive data on natural changes in the $\mathrm{X}-\mathrm{Y}$ inheritance exist for the platyfish, the housefly, and some other dipterans. In these organisms the genotypic structures of some multigenic mechanisms are known and each agrees with a model in the tables. There are also some data on variations in sex determining mechanisms for the midge, Chironomus tentans, (Thompson and Bowen, 1972) and some copepods (interpreted by Scudo, 1967), but the 
data are meagre and simply indicate that the differences between populations are due to few sex determiners.

Sex determination in the platyfish, Xiphophorus maculatus, has been extensively studied by Gordon (1946, 1954) and Kallman (1965, 1968, 1970, 1973). Kallman (1965) has shown that male and female heterogamety coexist in populations throughout most of its range. The genetics of sex determination are so simple that all genotypes are known: the genotypes are given by $\mathrm{lC}$, a single locus model. Unfortunately, the only data available on genotype frequencies are samples taken over a period of 20 years from one general locality (Kallman, 1973). The combined samples (0 XX, 2 FX, $85 \mathrm{FY}, 4 \mathrm{XY}, 24 \mathrm{YY}$ ) fit closely to an equilibrium in the neighbourhood of the FY-YY endpoint.

It is not known which condition is ancestral in the platyfish. The $\mathrm{F}$ and $\mathrm{X}$ are present in differing frequencies throughout the species' range and Kallman (1973) has suggested some reasons for the frequency distributions. Possibly, the XX/XY system is ancestral and the F is spreading because of a lack of sex-linked pigment genes (Kallman, 1970).

The data from the platyfish, therefore, support the theory in that (1) polygenic populations are known and (2) the genotypes behave according to one of the models. More extensive samples must be obtained before any rigorous comparison can be made between observed and expected genotype frequencies.

Three different mechanisms of sex determination are known in the housefly, Musca domestica. The details of these mechanisms are consistent with the models in this paper.

The most commonly observed mechanism in the housefly is male heterogamety in which the $\mathrm{X}$ and $\mathrm{Y}$ differ in size (Hiroyoshi, 1964; McDonald, 1971). In another mechanism both sexes are XX, and males are heterozygous for a male determining autosome III (Hiroyoshi, 1964; McDonald et al., 1975). One of these mechanisms has probably evolved from the other through the intermediate given by 3A. Populations with this multigenic mechanism are known from North America in localities geographically intermediate between the localities where the two simple mechanisms are found.

It is likely that the XY mechanism is ancestral to the others. The $\mathrm{X}$ and Y chromosomes exhibit heteropycnosis (Hiroyoshi, 1964), the Y is entirely heterochromatic (Jan and Shu, 1972), and the two are of unequal size. All these are characteristic of an old, well established sex chromosome mechanism. In the other two housefly mechanisms, the loci which determine sex are on an " autosome" which shows none of these characteristics. Therefore, we conclude that the evolution of $\mathrm{XX}$ males described above resulted when the $\mathrm{XX} / \mathrm{XY}$ mechanism was invaded by a strong male determiner on autosome III. For some reason, the XX males had higher fitness than the $\mathrm{XY}$ males, and the Y chromosome was selected against.

The evolution of XX males has occurred naturally at least once. Populations are found in Japan and North America in which a strong male determiner is found in autosome III (Hiroyoshi, 1964; McDonald, 1971). Cytological investigation has shown that there is no obvious Y-translocation to this autosome (Hiroyoshi, 1964; Bull and McDonald, unpublished observations on G-band analyses) so that the sex determiner may have been created by mutation. 
A second example of this change occurred in a cultured strain whose progenators were irradiated (Wagoner, 1968). In these animals the autosome IV was male determining due to translocation of part of the $\mathrm{Y}$ chromosome.

In the third housefly mechanism both sexes are again $\mathrm{XX}$, but females are heterozygous for a dominant female determiner (Wagoner, 1969; Wagoner and Johnson, 1974). The information on this system indicates that three loci have male determining alleles and one locus has a strong female determiner, but there are not sufficient data to permit a thorough analysis of all genotypes. However, since the female determiner is apparently dominant to all combinations of the male determiners at the other three loci (Wagoner, 1969), the system appears to be a straightforward extension of model 2A.

By analysis of the models it is evident that if this mechanism evolved from the $\mathrm{XY}$ mechanism, it did so in at least two steps, the first giving rise to $\mathrm{XX}$ males through the introduction of a strong male determiner (3A) or a mild male determiner (2B), and the second step being the invasion of the female determiner in this derived population. The alternatives of the first step are testable because the male determiner is preserved and increases in frequency in step 2. The three male determiners known are strong because $\mathrm{XX}$ is male. This suggests that model $3 \mathrm{~A}$, or an extension of it with two additional male genotypes, represents the ancestral population. However, the tests for male determiners would not have uncovered any mild male determiners, and therefore, model 2B cannot be ruled out.

Although the evolution and maintenance of the system above is too complex to analyse beyond this level, the occurrence of these sex determiners will permit the artificial construction of some of the populations given by the two-loci models. Model $2 \mathrm{~A}$ can be constructed using a marked " $\mathrm{X}$ " locus (autosome III), an unmarked male determining autosome III as the $\mathrm{Y}$, and the strong female determiner (above) as F. Populations could be initiated with different frequencies and be bred in discrete generations. The sampling would require only a single cross to determine genotypes.

A unique kind of sex determining mechanism is known in two other families of Diptera, the Phoridae (Mainx, 1964) and the Chironomidae (Beerman, 1955, discussed in Mainx, 1964). A single gene, M, determines maleness and varies in its position in the genome of males. Commonly, $\mathbf{M}$ is found at one of two loci (model 3A), but occasionally $\mathrm{M}$ is found at a third locus (model $3 \mathrm{~A}$ with an additional male genotype: $\mathrm{XX}$ bb $\mathrm{cM}$ ). What is most interesting is that a multigenic mechanism has arisen by translocation of $\mathbf{M}$ rather than a mutational origin of a new sex determiner.

The data from the dipterans are also supportive of the evolutionary models presented in this paper. At present, the only kind of multigenic mechanism known in detail is 3A. Since all possible genotype frequencies in this model are equilibrial, it is meaningless to assess congruence between expected and observed frequencies.

\section{Discussion}

The sex determining mechanisms of male and female heterogamety have evolved from common ancestors several times in animals. This paper has attempted to show how changes in the heterogametic mechanisms might take place. We suggest that male or female heterogamety may change because a mutant sex determiner which changes the inheritance of the $\mathrm{X}$ 
and $\mathrm{Y}$ invades a population. We constructed and analysed several single and two-loci models and observed that the mutant will successfully invade if some of the genotypes it creates have higher viability than the genotypes of the ancestral population $(\mathrm{XX} / \mathrm{XY})$.

The computer analyses of the models were limited to considering all genotypes of equal viability or all genotypes except one of equal viability. Many other viability combinations can be considered but they would reduce to special cases and be too numerous to present here. It seems likely, however, that the preliminary analyses of this study will provide the basic information about these models.

It is difficult to understand the exact behaviour of some of the models. For many genotypes, the frequency in one generation is dependent upon the frequencies of several mating combinations from the previous gcnerations, as in Hardy-Weinberg systems. In addition, most of the models are compounded by sex ratio selection. Sex ratio selection for autosomal genes will act to maintain equal numbers of the two sexes at conception in these models (Fisher, 1930; Shaw, 1958; Eshel, 1975). In some of the models there are mating combinations that do not produce equal numbers of males and females. If an inequality results in the total numbers of the two sexes at conception, then the genotypes which produce a bias of the rarer sex may be at an advantage. However, it is difficult to understand how sex ratio selection acts in these models because they deal with sex linked genes. Sex ratio selection for autosomal genes differs from that for sex linked genes (Shaw, 1958). It is therefore interesting that the sex ratios of the neutral equilibria are always $1 / 2$.

An invading sex determining mutant faces no disadvantage from sex ratio selection. It will spread if it gives rise to genotypes of only slightly higher viability than the original genotypes, bbXX and bbXY. However, there are few obvious biological reasons why an invading sex determiner would evcr have an advantage over the existing ones. Possibly the fitness of a sex determiner depends at least partly upon the alleles at adjacent loci. Alleles very close to the sex determiners will be tightly linked, and thus affect the fitness of the sex determiner also. Kallman $(1970,1973)$ has proposed this as a means of selection in the platyfish populations. The sex determining alleles are often linked with traits for colour patterns, being advantageous in the male but not the female. The $\mathrm{F}$ allele, unlike the $\mathrm{X}$, lacks closely linked pigment traits and may be an advantage to this sex determiner.

Distorting the segregation of the alleles in a genotype affects the dynamics of the models. This was suggested to us by J. Maynard Smith (pers. comm.). Segregation distortion differs from the kind of fitness manipulations described above. In some preliminary analyses we observed that segregation distortion in the bbXY genotype either favoured the mutant, in some models, or selected against it. If the mutant was favoured, then the population evolved to a stable, internal equilibrium in some models, or evolved to fixation of the new recurrent pair. The sex ratio at equilibrium was often different from 1/2, and there was no indication that selection was acting in these populations to evolve toward a population with a sex ratio of $1 / 2$. Segregation distortion may be a common occurrence in natural populations and therefore offers a likely means by which a mutant sex determiner may become established.

Winge proposed that the change in $\mathrm{X}-\mathrm{Y}$ inheritance was brought about 
by the combination of weak sex determiners at many loci. This in contrast to our models which deal with only a single or two-loci. We have already discussed several examples which support the models here, but it would be interesting to know if Winge's model is valid in some cases. At present it is difficult to know. Although some other fish geneticists have also advocated Winge's model (Oktay, 1959; Yamamoto, 1963), Kallman (1965, pp. 173178) has carefully reanalysed the data and concludes that there is no good evidence in favour of Winge's hypothesis; the data from the platyfish (one of several species studied) even indicate the opposite: sex reversals are due to few autosomal sex determiners (Kallman, 1965).

Comparison of the models to data from natural populations has shown that the general concept used in this paper is probably valid, even though the comparison is based on few observations. We, therefore, use the models to interpret some general patterns among sex determining mechanisms. For example, the models demonstrate how the different systems of genic balance may arise. Bridges' insight was that the autosomes can play a role in sex determination. Thus, only the $\mathrm{X}$ or the $\mathrm{Y}$ need be sex determining, and it is often the case that only one of the sex chromosomes has a noticeable sex determining influence (White, 1973). The two-loci models demonstrate how sex determiners can become autosomally inherited and, simultaneously, demonstrate how a heterogametic mechanism can evolve in which only one of the two alleles at the new locus of heterozygosity is sex determining.

Sex chromosomes will be less subject to change than heterogametic systems of a single locus. The degenerate nature of $\mathrm{Y}$ chromosomes prevents the establishment of any sex determiner that gives rise to YY genotypes. Therefore, in organisms with a degenerate $\mathrm{Y}$ chromosome, only those changes giving rise to $\mathrm{XX}$ males should occur. Also, large $\mathrm{X}$ chromosomes may carry genes (other than sex determiners) which selection has favoured because of their single dose in males $(\mathrm{XY})$ and double dose in females (XX). Resuming autosomal inheritance of the $\mathrm{X}$ might be disadvantageous because of the double dose that males would receive.

Acknowledgments.-We thank Drs Klaus Kallman of the New York Aquariums and Ian McDonald of the U.S.D.A. in Fargo, North Dakota, for collaboration on various parts of the manuscript. Ian McDonald was especially helpful in providing information about the housefly. Dr Russell Bjorkland of the University of Utah provided a critical mathematical insight which we gratefully acknowledge. We thank Dr William Coles of the University of Utah for helpful discussions on the mathematical solutions to some of the models. Dr Graham Pyke provided helpful comments on earlier versions of the paper.

\section{References}

BACCI, G. 1965. Sex Determination. Pergamon Press, Oxford.

BAteman, A. J. 1952. Self-incompatibility systems in angiosperms. I. Theory. Heredity, 6 , 285-310.

BEERMANN, w. 1955. Geschlechtsbestimmung und evolution der genetischen Y-Chromosomen bei Chironomus. Biol. Zentr., 74, 525-544 (cited in Mainx, 1964).

BRIDGES, c. B. 1925. Sex in relation to chromosomes and genes. Am. Nat., 59, 127-137.

CANNINGS, C. 1968. Fertility differences between homogamous and heterogamous matings. Genet. Res., 11, 289-301.

ESHEL, I. 1975. Selection on sex ratio and evolution of sex determination. Heredity, 34, 351-361.

EWENS, W. J. 1964. On the problem of self-sterility alleles. Genetics, 50, 1433-1438.

FALK, C., AND LI, C. C. 1969. Negative assortative mating: exact solution to a simple model. Genetics, 62, 215-223. 
FINNEY, D. J. 1952. The equilibrium of a self-incompatible polymorphic species. Genetica, $26,33-64$.

FISHER, R. A. 1930. The genetical theory of natural selection. Dover Reprint (1958), New York.

FISHER, R. A. 1941. The theoretical consequences of polyploid inheritance for the mid-style form of Lythrum salicaria. Ann. Eugenics, II, 31-38.

GoldschmidT, R. B. 1934. Lymantria. Bibliogr. Genet., 11, 1-180.

GORDON, м. 1946. Interchanging mechanisms for sex determination for fishes under domestication. F. Heredity, 37, 307-320.

GORDON, M. 1954. Two opposing sex determining mechanisms, one XX-XY, and the other WY-YY in different natural populations of the platyfish, Xiphophorus maculatus. Proc. 9th Int. Cong. Gen., 2, 960-964. (Supplement to Caryologia, v. 6).

hiroyoshi, т. 1964. Sex limited inheritance and abnormal sex ratio in strains of the housefly. Genetics, 50, 373-385.

JAN, K. Y., AND SHU, ANN-PING. 1972. Localization of repetitive DNA in housefly chromosomes with a modified Giemsa stain. Bull. Inst. Zool. Acad. Sinica, 11 (2), 29-33.

KALLMAN, K. D. 1965. Genetics and geography of sex determination in the poeciliid fish, Xiphophorus maculatus. Zoologica, 50, 151-190.

KALLMAN, K. D. 1968. Evidence for the existence of transformer genes for sex in the teleost Xiphophorus maculatus. Genetics, 60, 811-828.

Kallman, K. D. 1970. Sex determination and the restriction of sex-linked pigment patterns to the $\mathrm{X}$ and $\mathrm{Y}$ chromosomes in populations of a poeciliid fish, Xiphophorus maculatus, from the Belize and Sibun rivers of British Honduras. Zoologica, 55, 1-16.

Kallman, K. D. 1973. The sex determining mechanism of the platyfish, Xiphophorus maculatus. In Genetics and Mutagenesis of Fish, ed. J. H. Schröder. Springer-Verlag, Berlin.

KARLin, s., AND FELDMAN, M. w. 1968a. Analysis of models with homozygote $\times$ heterozygote matings. Genetics, 59, 105-116.

KARLIN, s., AND FELDMAN, M. w. 1968b. Further analysis of negative assortative mating. Genetics, 59, 117-136.

Kosswig, c. 1964. Polygenic sex determination. Experientia, 20, 190-199.

MCDONALD, I. 1971. A male-producing strain of the housefly. Science, 172, 489.

MCDONALD, I., OVERLAND, D. E., LeOpold, R. A., DEGRUGILleR, M. E., MORGAN, P. B., AND hoffman, н. c. 1975. Genetics of houseflies. F. Heredity, 66, 137-140.

mainx, F. 1964. The genetics of Megaselia scalaris Loew (Phoridae): A new type of sex determination in Diptera. Am. Nat., 98, 415-430.

martin, J. 1966. Female heterogamety in Polypedilum nubifer (Diptera: Nematocera). Am. Nat., 100, 157-159.

oxtAy, M. 1959. Uber ausnahmemaennchen bei Platypoecilus maculatus und eine neue Sippe mit XX-maennchen und XX-weibohen. Istanbul Univ. Fen Fak. Mecm., B 24, 75-91 (as cited in Kallman, 1965).

scudo, F. M. 1964. Sex population genetics. Ri. Sci., 34, II-B, 93-146.

scudo, F. м. 1967. Criteria for the analysis of multifactorial sex determination. Mon. Zool. Ital., $1,1-21$.

SHAW, R. F. 1958. The theoretical genetics of the sex ratio. Genetics, 43, 149-163.

THOMPSON, P. E., AND BOWEN, J. s. 1972. Interactions of differentiated primary sex factors in Chironomus tentans. Genetics, 70, 491-493.

WAGONER, D. E. 1968. Linkage group-karyotype correlation in the housefly, Musca domestica $\mathrm{L}$., confirmed by cytological analysis of $\mathrm{X}$-ray induced $\mathrm{Y}$-autosome translocations. Genetics, 62, 115-121.

WAGONER, D. F. 1969. Presence of male determining factors found on three autosomes in the housefly, Musca domestica. Nature, 223, 187-188.

WAGONFR, D. E., AND JOHNSON, O. A. 1974. Strains of house flies producing only males. Ann. Ent. Soc. Am., 67, 553-554.

white, M. J. D. 1973. Animal Cytology and Evolution, 3rd Ed. Cambridge University Press, London.

wINGE, O. 1932. The nature of sex chromosomes. Proc. 6th Int. Congr. Genet., 1, 343-355.

WINGE, o. 1934. The experimental alteration of sex chromosomes into autosomes and vice versa, as illustrated by Lebistes. Compt. rend. Lab. Carlsberg. Ser. Physiol., 21, 1-49.

wiNGE, O. 1937. Goldschmidt's theory of sex determination in Lymantria. F. Genetics, 34, 81 89.

уамамото, $\mathrm{T}$. 1963. Induction of sex reversal in sex differentiation of YY zygotes in the medaka, Oryzias latipes. Genetics, 48, 293-306. 